\title{
Carotid atherosclerosis promotes the progression of Alzheimer's disease: A three-year prospective study
}

\author{
JING XIANG ${ }^{1-3}$ \\ ${ }^{1}$ Criminal Investigation College, Southwest University of Political Science and Law; ${ }^{2}$ Chongqing Institutes \\ of Higher Education Key Forensic Science Laboratory, Chongqing Institutes of Higher Education Center \\ of Forensic Science Engineering and Research, Chongqing 401120; ${ }^{3}$ Department of Neurology, \\ Daping Hospital, The Third Military Medical University, Chongqing 400042, P.R. China
}

Received April 25, 2016; Accepted March 17, 2017

DOI: 10.3892/etm.2017.4661

\begin{abstract}
Although cerebrovascular diseases have been considered as risk factors for cognitive decline and dementia, the associations between atherosclerosis and Alzheimer's disease (AD) have not been fully examined and remain controversial. The aim of this three-year prospective study was to investigate whether arotid artery atherosclerosis accelerates cognitive impairment in AD patients. The association of carotid intimal medial thickness (IMT) with prospective trajectories of cognitive function was assessed among 521 patients with light to moderate AD, and 437 AD patients were followed up annually for 3 years. Participants underwent initial carotid ultrasonography and repeated neuropsychological testing every year. Mixed-effects regression analyses were adjusted to estimate the effect of carotid IMT and other adjusting variables. The results of the present study indicated that carotid IMT was significantly associated with various measures of cognitive function. Furthermore, AD patients with higher carotid IMT values had a faster decline in cognitive scores in a variety of neuropsychological tests, particularly in verbal and non-verbal memory, semantic fluency and executive function. The present prospective study showed that carotid atherosclerosis is a predictive factor regarding the progression of cognitive impairment in AD patients, suggesting that early detection and treatment of vascular risk factors may prevent or at least postpone the evolution of the disease.
\end{abstract}

\section{Introduction}

Alzheimer's disease (AD), the most common form of dementia, is a progressive neurodegenerative disease characterized by

Correspondence to: Dr Jing Xiang, Criminal Investigation College, Southwest University of Political Science and Law, 301 Baosheng Avenue, Huixing, Chongqing 401120, P.R. China E-mail: jingxiang129@sina.com

Key words: carotid intimal medial thickness, Alzheimer's disease, progression, cognitive impairment, atherosclerosis, neuropsychology a progressive decline in cognitive function, endangering a growing proportion of the elderly population (1). Due to the lack of effective treatments, the search for means of preventing/delaying the onset/progression of $\mathrm{AD}$ remains a major research area (2). Exploration of methods for adjusting risk factors is one direction to pursue in preventing/delaying AD. Epidemiological studies have revealed that vascular factors are involved in the increase of the incidence of $\mathrm{AD}$, possibly resulting in poor prognosis $(3,4)$.

In general, vascular disease frequently coexists with degenerative intracranial changes of patients with dementia, which makes it difficult to identify risk factors associated with cognitive decline and diagnose types of dementia. In a population-based study on 308 patients with dementia, types of dementia were re-evaluated in combination with data on vascular lesions, as well as the incidence and progression of the disease (5). Only $47 \%$ of those patients initially diagnosed with $\mathrm{AD}$ were reclassified as pure AD, only $25 \%$ of those diagnosed with vascular dementia (VD) were reclassified as pure VD and most of the cases were of mixed dementia. There has been a growing interest in the hypothesis that vascular diseases are associated with AD and certain studies have revealed that vascular system dysfunction may trigger the amyloid cascade $(6,7)$.

A previous study also found that vascular risk factors accelerate the progression of AD (8). A consensus has now been reached that early detection and treatment of classic vascular risk factors reduce their impact on the epidemiology of dementia $(9,10)$. Although intimal medial thickness (IMT), a marker of early atherosclerosis, is associated with cognitive decline and can be used to measure subclinical vascular disease (11), previous studies have mainly focused on community population samples with or without a history of stroke (12-14). A longitudinal study on 66 patients with AD showed a significant association between IMT and the progression of cognitive impairment at 12-month follow-up (15). Although an increasing number of studies investigated the association between vascular factors and cognitive impairment in patients with $\mathrm{AD}$, the findings showed rather inconsistent and weak evidence of the association (16). Previous studies, most of which were cross-sectional with a relatively small sample size, showed a weak association between IMT and 
cognitive impairment, whereas a few longitudinal studies revealed a rather significant association $(17,18)$. The lack of longitudinal research design with a relatively large sample calls for a large sample panel study on the association of vascular factors and cognitive impairment in patients with AD. The present three-year prospective study sought to establish whether carotid artery atherosclerosis has a considerable impact on the acceleration of the progression of cognitive decline in Chinese patients with early-stage AD, with a focus on identifying the possible follow-up treatment, i.e., surgical procedure or medication.

\section{Materials and methods}

Study population. As the present study was a three-year panel study, data were initially collected for 521 patients randomly selected from 7 urban communities in the Chongqing metropolitan area (total population, 35 million in Chongqing municipality, including 10 million urban residents), 437 of which remained after the three-year follow-up. The sample was selected on the basis of the following criteria: i) Diagnosis with probable $\mathrm{AD}$ according to the National Institute of Neurological and Communicative Disorders and Stroke and the Alzheimer's Disease and Related Disorders Association criteria (19); and ii) mild to moderate phase of the disease: 1-2 on the Clinical Dementia Rating (CDR) scale (20). The exclusion criteria were as follows: i) Clinical history of cerebrovascular diseases, focal neurological signs, severe subcortical leukoencephalopathy and/or cortical infarction (21); ii) a Hachinski score (22) of $>4$; iii) a diagnosis of other types of dementia; iv) inability to comply with the study assessment; v) mental illness; vi) alcohol or drug abuse; and vii) relocation from the area or refusal to participate (8).

The clinical diagnosis of AD was made by a group of highly qualified neurologists and psychiatrists. The present study was approved by the Institutional Review Board of Third Military Medical University (Chongqing, China) and all subjects provided informed written consent according to the Declaration of Helsinki.

Clinical assessment. On admission, all patients underwent a structured interview for demographic data and medical history, as well as a neurological examination and head imaging. The following data were prospectively collected: Age, sex, educational level, cigarette smoking, hypertension (previously diagnosed and treated or systolic pressure $>160 \mathrm{mmHg}$ and/or diastolic pressure $>90 \mathrm{mmHg}$ persistently observed during admission after the acute phase), diabetes mellitus (previously diagnosed and treated or fasting glucose $>7 \mathrm{mmol} / \mathrm{l}$ in two blood samples after the acute phase), and atrial fibrillation (diagnosed and treated previously or during admission). All patients underwent magnetic resonance imaging (MRI), brain scans as well as fluid-attenuated inversion recovery (FLAIR). Among them, MRI sequences were used to detect infarction and cerebral white matter lesions. Cerebral white matter lesions were also defined as ill-defined periventricular hyper-intense signals on T2 or FLAIR phase width an area of area $\geq 5 \mathrm{~mm}$. Lesions were quantitatively rated using the Wahlund scale (23).
Neuropsychological evaluation. Cognitive status was assessed using the Chinese version of the Mini-Mental Status Examination (CMMS), which was previously validated in elderly Chinese individuals as cognitive screening measures (24), with a scale ranging from 0-30 and with the boundary score of CMMS defined as 17 (illiteracy), 20 ( $\leq 6$ years of education) and 24 (>6 years of education). Subjects who were abnormal according to CMMS assessment were subjected to further neuropsychological tests, including Alzheimer Disease Assessment Scale-cognitive subscale (ADAS-cog) (25), CDR (20), Fuld Object Memory Evaluation (FOM) (26), Rapid Verbal Retrieve (RVR) (27), the Wechsler Adult Intelligence Scale [Digit Span (DS) and Block Design (BD) subtests] (28).

Functional ability to perform basic and instrumental activities was evaluated using a modified Activities of Daily Living (ADL) test (29), which contained 20 basic and instrumental activities of daily living. Each activity was rated on a 4-point scale: 1 , no limitation; 2 , performs activities by oneself with certain difficulties; 3 , requires help with activities; and 4, unable to perform any activity. Additive scores of ADL ranged from 20 to 80 , with an ADL score $>20$ indicating that the subject had certain difficulties in self-care. The Hachinski Ischemic Scale (22) was used for evaluating significant vascular disease.

Ultrasound examination. A high-resolution B-mode ultrasound examination of the left and right carotid arteries was performed on 437 subjects using an Aloka SSD-650 (Hitachi, Tokyo, Japan) with a 7.5- and 10-MHz transducer. Acquisition, processing and storage of B-mode images were computer-assisted, utilizing the software specifically designed for longitudinal studies (30). The examination involved scanning the common carotid arteries, the carotid bifurcations and the first $2 \mathrm{~cm}$ of the internal carotid arteries. The IMT (31) (distance between the media-adventitia interface and the lumen-intima interface) was automatically measured twice on longitudinal B-mode images of the far wall of each carotid artery. The value assigned corresponded to the maximum thickness of the intima media of the carotid arteries. Most of the examinations were performed by one examiner and all analyses of IMT were made by the same skilled observer.

In a previous study, the association between carotid artery stenosis and cognitive impairment was explored in a Chinese population, revealing that the IMT value has more a significant association with cognitive impairment than stenosis (32), leading to a major focus on the IMT rather than stenosis in the present study.

Follow-up. Trained neurologists and psychiatry experts were performed annual follow-up from November 2011 to November 2014. Each follow-up procedure included CMMS, ADAS-cog, ADL, RVR, BD, DS and FOM assessment. If new neurological signs of localization suggested new brain lesions, a cranial MRI scan was performed.

Statistical analysis. Statistical analysis was performed using SPSS 19.0 for Windows (International Business Machines Corp., Armonk, NY, USA). All variables including adjusting factors (e.g., age, sex, education and vascular risk factors) as well as carotid IMT were introduced into mixed-effect 
regression analyses for the three-year panel data in order to investigate longitudinal associations of carotid IMT with cognitive function. Mixed-effect regression adopts fixed as well as random effects in the estimation and eliminates the effects of confounding variables from unmeasured time-invariant causes in the panel data (33). In the mixed-effects regression analyses, each measure of cognitive function was separately regressed on carotid IMT (i.e., overall differences in carotid IMT over the three-year period), a battery of adjusting variables, and an interaction term of carotid IMT with age (i.e., changes in cognitive functions associated with carotid IMT over time). Values are expressed as the mean \pm standard deviation (SD).

\section{Results}

The study included 521 patients with mild to moderate AD, among which 45 patients did not comply with the research assessment, 33 patients were lost to follow-up and 6 patients died, while the 437 patients remaining successfully completed the 3-year follow-up study. The mean age of the subjects at baseline was $69.2 \pm 10.5$ years, $45.6 \%$ were male and the mean education was 10.7 years. The mean carotid IMT was $2.3 \pm 1.2 \mathrm{~mm}$ (range, 0.8-4.6 mm). Demographic data and the clinical baseline characteristics are presented in Table I.

First, mixed-effects regression analysis for CMMS was performed in order to estimate the effect of carotid IMT and other adjusting variables. As expected, the preliminary mixed-effects regression estimate of the model (not presented here) did not reveal any statistically significant effect of carotid IMT on CMMS. In the subsequent mixed-effects regression estimation (Table II), a series of neuropsychological and functional test scores were regressed on the carotid IMT value and other adjusting variables in all six models. The results showed that carotid IMT had a statistically significant effect in four of the models (ADAS-cog, FOM, DS, and RVR), while the effect of carotid IMT was not significant in the ADL and BD models. It was also found that the effects of the interaction between carotid IMT and age (change over time) were statistically significant in ADAS-cog $(b=-0.676, P=0.036)$, FOM $(b=-0.41, P=0.023)$, DS ( $b=-1.35, P=0.034)$ and RVR $(b=-1.52$, $\mathrm{P}=0.038$ ), respectively.

Figs. 1-4 present significant carotid IMTxAge interactions for ADAS-cog, ADL, DS and RVR, respectively, and visualize the nature of the significant interactions between carotid IMT and age in the four regression models in Table II. Each fig. shows age-associated changes in cognitive performance as a function of different levels of carotid IMT (IMT=1.1, 2.3 and $3.5 \mathrm{~mm}$ ), with all the covariates adjusted in the analyses. The figures are based on the association of mean value of carotid $\mathrm{IMT} \pm \mathrm{SD}$ and predictive cognitive values. The figures clearly show that the higher the IMT value, the steeper the decline in the four neuropsychological test scores as people age.

\section{Discussion}

The present three-year prospective study investigated the longitudinal change of cognitive function in patients with mild to moderate AD in terms of different carotid artery IMT values. During the follow-up period, patients with a relatively high IMT showed a significantly greater prospective decline
Table I. Baseline characteristics of patients with mild to moderate Alzheimer's disease.

\begin{tabular}{lcc}
\hline Variables & Mean (SD) & Range \\
\hline Age (years) & $69.2(10.5)$ & $58-88$ \\
Gender (\% male) & 45.6 & \\
Education (years) & $10.7(3.5)$ & $6-17$ \\
Mean arterial pressure (mmHg) & $97.3(14.8)$ & $64-165$ \\
Total cholesterol (mg/dl) & $201.4(32.6)$ & $115-323$ \\
Body mass index $\left(\mathrm{kg} / \mathrm{m}^{2}\right)$ & $25.3(4.2)$ & $16.7-38.4$ \\
Cardiovascular medication use (\%) & 19.8 & \\
Current smoking habit $(\%)$ & 45.1 & \\
Hachinski ischemic scale & $2.3(1.2)$ & $0-5$ \\
Wahlund scale & $4.6(4.5)$ & $0-14$ \\
Mean carotid IMT (mm) & $2.3(1.2)$ & $0.8-4.6$ \\
\hline
\end{tabular}

SD, standard deviation; IMT, intimal medial thickness.

Table II. Mixed-effects regression estimates of neuropsychological test results and performance on carotid IMT and covariates.

\begin{tabular}{|c|c|c|c|c|}
\hline \multirow[b]{2}{*}{ Neuropsychological test } & \multicolumn{2}{|c|}{$\begin{array}{c}\text { Carotid } \\
\text { IMT }\end{array}$} & \multicolumn{2}{|c|}{$\begin{array}{c}\text { Carotid } \\
\text { IMTXAge }\end{array}$} \\
\hline & $\mathrm{b}$ & SE & $\mathrm{b}$ & SE \\
\hline ADAS-cog & $-35.2^{\mathrm{a}}$ & 14.3 & $-0.68^{\mathrm{b}}$ & 0.31 \\
\hline ADL & 1.46 & 0.98 & -0.03 & 0.01 \\
\hline FOM & $-23.5^{\mathrm{a}}$ & 11.7 & $-0.41^{\mathrm{b}}$ & 0.18 \\
\hline $\mathrm{BD}$ & -17.3 & 24.2 & -1.32 & 0.08 \\
\hline DS & $-46.2^{\mathrm{a}}$ & 29.2 & $-1.35^{\mathrm{b}}$ & 0.53 \\
\hline RVR & $-65.2^{\mathrm{a}}$ & 32.4 & $-1.52^{\mathrm{b}}$ & 0.52 \\
\hline
\end{tabular}

${ }^{\text {a }} \mathrm{P}<0.05$ vs. effect of Carotid IMT. ${ }^{\text {b }} \mathrm{P}<0.05$ vs. Carotid IMTxAGE. ADAS-cog, Alzheimer's Disease Assessment Scale-cognitive subscale; ADL, Activities of Daily Living; FOM, Fuld Object Memory evaluation; BD, Block Design; DS, Digital Scale; RVR, Rapid Verbal Retrieve; IMT, intimal medial thickness; b, coefficient; SE, standard error.

in performance according to various cognitive measures, including ADAS-cog, RVR, FOM and DS. Individuals with a greater carotid IMT showed a faster prospective decline in cognitive function, particularly in verbal fluency and memory for objects and digits. The present findings are consistent with previous ones, according to which cognitive decline in AD patients was associated with subclinical vascular disease (15).

The results of the present study demonstrated that carotid IMT is associated with a various types of cognitive dysfunction, particularly verbal and non-verbal memory, semantic fluency and executive function. Among the scales significantly associated with IMTXAge, FOM is a group of neuropsychological tests including memory, physical perception (touch discrimination), 


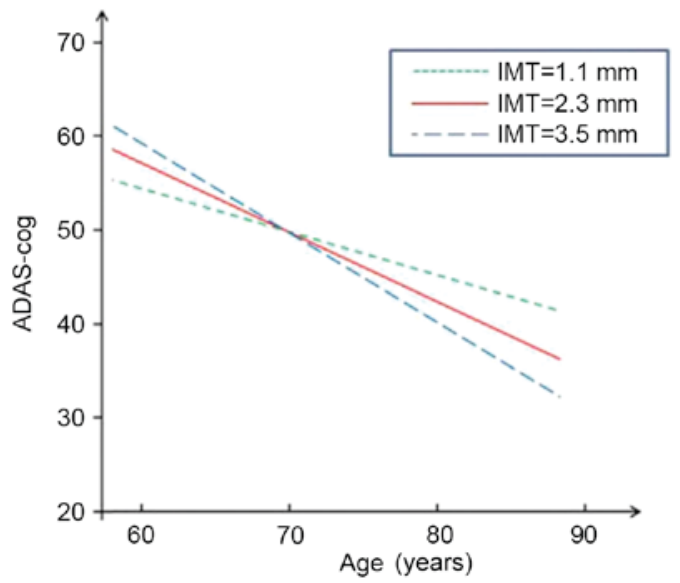

Figure 1. Longitudinal change in performance on ADAS-cog. IMT, intimal medial thickness; ADAS-cog, Alzheimer's Disease Assessment Scale-cognitive subscale.

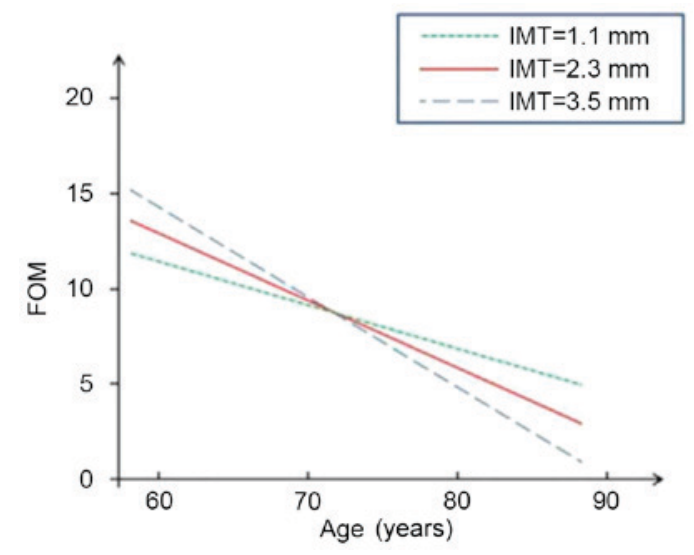

Figure 2. Longitudinal change in performance on FOM. FOM, Fuld Object Memory evaluation; IMT, intimal medial thickness.

visual naming, left-right orientation and learning features, while RVR is applied to detect semantic memory storage function. FOM and RVR can be used in combination to evaluate the degree of memory impairment. In the present study, DS was adopted from the Wechsler intelligence tests, and immediate memory and attention are major measures of cognitive function in this test. By combining a battery of neuropsychological tests, detailed information on cognitive function in verbal and non-verbal memory, semantic fluency and executive function was obtained.

Previous studies suggested several hypothetical interpretations of the association between vascular risk factors and AD. While it has been speculated that cerebrovascular disease is not involved in an account of cerebral atrophy caused by neurodegeneration, it has also been suggested that cerebral vascular factors have a key role in inducing or maintaining the pathological process of typical AD degeneration (34). Other studies reported that a lack of cerebral blood flow may amplify or strengthen the influence of the degeneration process, independently causing additional damage (35). It was also suggested that chronic cerebral hypoperfusion caused by severe carotid artery atherosclerosis is one of the causes of

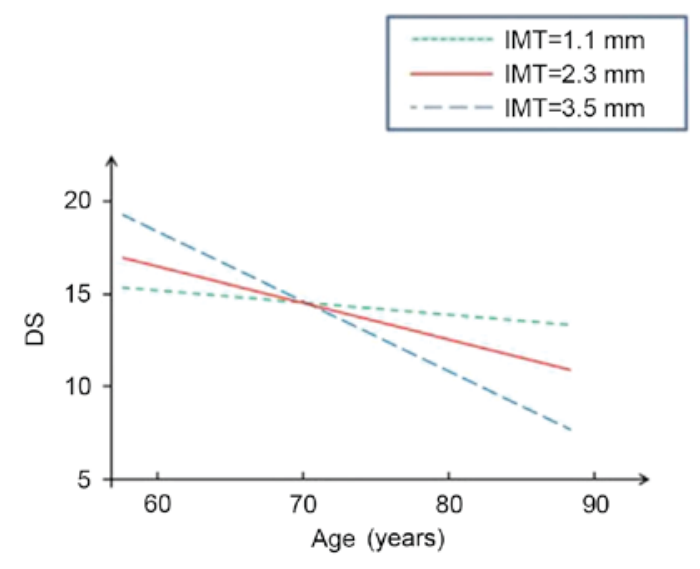

Figure 3. Longitudinal change in performance on DS. IMT, intimal medial thickness; DS, Digital Scale.

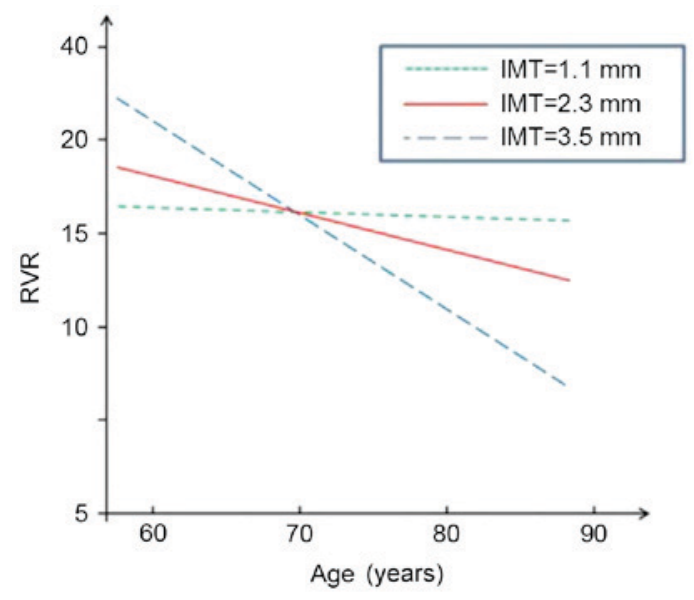

Figure 4. Longitudinal change in performance on RVR. IMT, intimal medial thickness; RVR, Rapid Verbal Retrieve.

cognitive impairment (36). In a previous study, in which cerebrovascular pathology appears to be, along with aging, a key permissible factor for the development of dementia-associated neurodegeneration, it was found that AD patients showed a significantly higher prevalence of vascular pathology and cerebrovascular disease than patients with $\alpha$-synucleinopathy, prion disease and frontotemporal lobar degeneration due to immunoreactive inclusions of Tau and TAR DNA binding protein 43 (37).

A previous study suggested that vascular risk factors are associated with mild cognitive impairment and AD (38). Previous findings regarding VD and AD patients also showed that vascular risk factors, brain pathology and cognitive characteristics have a greater overlap than previously known (39), and particularly individuals with memory impairment have a risk of various types of dementia. Moreover, it has been proposed that atherosclerosis and $\mathrm{AD}$ share a common infectious/inflammatory pathoetiology and individuals with atherosclerosis or AD are neither merely 'ill' nor 'old', but that acquired infection/inflammation and endocrine aging are rather likely to have a joint role in causing these age-associated diseases (40). Subclinical vascular diseases may directly or indirectly affect cognitive function through 
various mechanisms, and the measured values of carotid atherosclerosis are associated with various cardiovascular risk factors, including demographic, metabolic, immune and lifestyle factors as well as cognitive decline (41). Other hypothetical mechanisms are common genetic susceptibility (such as apolipoprotein E $\varepsilon 4$ allele), chronic cerebral hypoperfusion, asymptomatic cerebral infarction and other associated brain structural changes, such as atrophy of the cortex (42).

The present study clearly indicated that the severity of carotid artery atherosclerosis accelerates cognitive impairment and acts as a potential risk factor for AD. Loss of cognition allegedly has a detrimental influence on individuals and society, calling for active prevention or deceleration of its progress. Considering the fact that the development of any specific disease-modifying treatment for AD is unlikely in the near future, the present study underscores the possibility that early intervention to reduce atherosclerosis may be of clinical benefit for AD. It further suggests that the application of various treatments for carotid arterial atherosclerosis is worthwhile in order to assess their effect on cognitive function in patients.

Considering the difficulty of identifying the respective contributions of degenerative and vascular components to morbidity and pathological processes in each patient, a more rational approach to the problem may be a comprehensive and consistent treatment to keep modifiable vascular risk factors at their lowest level among patients with cognitive impairment, regardless of whether they have been diagnosed as dementia (43). A previous study revealed that the treatment of vascular risk factors may slow down cognitive decline in patients with AD (44). However, it has rarely been reported whether treatments of IMT, such as interventional operation, decelerate the progression of AD. The present study confirmed that severe IMT greatly accelerates the progress of AD, further suggesting that AD patients with high IMT may benefit from aggressive endovascular treatment.

For the prevention and treatment of $\mathrm{AD}$, it is important to make a diagnosis as early as possible and to intervene with risk factors that are pivotal for its progression (45). In either normal or cognitively impaired population groups, asymptomatic carotid atherosclerotic disease has an important role in accelerating cognitive damage (46). It is noteworthy that the present study was performed in a large Chinese metropolitan area with $93 \%$ of the population being of Han Chinese ethnicity who have similar cultural habits, including a relatively hig-fat diet, which represents a risk factor for atherosclerosis.

In conclusion, while certain controversies remain regarding the association between vascular factors and cognitive impairment in patients with $\mathrm{AD}$, the present 3-year prospective study provided further evidence that carotid atherosclerosis is a predictive or predisposing factor for the progression of cognitive impairment in AD patients, particular that regarding verbal and non-verbal memory, semantic fluency and executive function. The combined application of widely used neuropsychological measurement scales and the use of time-varying covariates are highlighted in this study. However, the limitations of the present study were that only patients with mild to moderate AD were selected as the study sample, and that the carotid artery IMT was used as a single parameter to resemble carotid atherosclerosis in the assessment. In addition, it should be noted that among previous studies, the use of inconsistent study samples as well as different follow-up periods and neuropsychological scales may also lead to the contradictory and inconsistent findings in this field. Therefore, the association of carotid artery IMT with the accelerated decline of cognitive function and even other types of cognitive impairment should be further studied after adjusting these covariate factors.

\section{Acknowledgements}

Funding for this study was provided by the Program for Innovation Team Building at Institutions of Higher Education in Chongqing (grant no. KJTD201301).

\section{References}

1. Breteler MM: Vascular risk factors for Alzheimer's disease: An epidemiologic perspective. Neurobiol Aging 21: 153-160, 2000.

2. Zhu CW, Scarmeas N, Torgan R, Albert M, Brandt J, Blacker D, Sano M and Stern Y: Longitudinal study of effects of patient characteristics on direct costs in Alzheimer disease. Neurology 67: 998-1005, 2006.

3. Gorelick PB: Risk factors for vascular dementia and Alzheimer disease. Stroke 35 (11 Suppl 1): S2620-S2622, 2004.

4. Viticchi G, Falsetti L, Vernieri F, Altamura C, Bartolini M, Luzzi S, Provinciali L and Silvestrini M: Vascular predictors of cognitive decline in patients with mild cognitive impairment. Neurobiol Aging 33: 1127.e1-9, 2012.

5. Aguero-Torres H, Kivipelto M and von Strauss E: Rethinking the dementia diagnosis in a population-based study: What is Alzheimer's disease and what is vascular dementia? A study from the Kungsholmen project. Dement Geriatr Cogn Disord 22: 244-249, 2006

6. Grammas P, Yamada M and Zlokovic B: The cerebromicrovasculature: A key player in the pathogenesis of Alzheimer's disease. J Alzheimers Dis 4: 217-123, 2002.

7. Humpel $\mathrm{C}$ and Marksteiner J: Cerebrovascular damage as a cause for Alzheimer's disease. Curr Neurovasc Res 2: 341-347, 2005.

8. Li J, Zhang M, Xu ZQ, Gao CY, Fang CQ, Deng J, Yan JC, Wang YJ and Zhou HD: Vascular risk aggravates the p rogression of Alzheimer's disease in a Chinese cohort. J Alzheimers Dis 20: 491-500, 2010.

9. Luzzi S, Vella L, Bartolini M, Provinciali L and Silvestrini M: Atherosclerosis in the evolution of Alzheimer's disease: Can treatment reduce cognitive decline? J Alzheimers Dis 20: 893-901, 2010.

10. López-Olóriz J, López-Cancio E, Arenillas JF, Hernández M, Jiménez M, Dorado L, Barrios M, Soriano-Raya JJ, Miralbell J, Cáceres C, et al: Asymptomatic cervicocerebral atherosclerosis, intracranial vascular resistance and cognition: The AsIA-neuropsychology study. Atherosclerosis 230: 330-335, 2013.

11. Lorenz MW, Markus HS, Bots ML, Rosvall M and Sitzer M: Prediction of clinical cardiovascular events with carotid intima-media thickness: A systematic review and meta-analysis. Circulation 30: 459-467, 2007.

12. Johnston SC, O'Meara ES, Manolio TA, Lefkowitz D, O'Leary DH, Goldstein S, Carlson MC, Fried LP and Longstreth WT Jr: Cognitive impairment and decline are associated with carotid artery disease in patients without clinically evident cerebrovascular disease. Ann Intern Med 140: 237-247, 2004.

13. Komulainen P, Kivipelto M, Lakka TA, Hassinen M, Helkala EL, Patja K, Nissinen A and Rauramaa R: Carotid intima-media thickness and cognitive function in elderly women: A population-based study. Neuroepidemiology 28: 207-213, 2007.

14. Muller M, Grobbee DE, Aleman A, Bots $M$ and van der Schouw YT: Cardiovascular disease and cognitive performance in middle aged and elderly men. Atherosclerosis 190: 143-149, 2007.

15. Silvestrini M, Gobbi B, Pasqualetti P, Bartolini M, Baruffaldi R, Lanciotti C, Cerqua R, Altamura C, Provinciali L and Vernieri F: Carotid atherosclerosis and cognitive decline in patients with Alzheimer's disease. Neurobiol Aging 30: 1177-1183, 2009. 
16. Urbanova B, Tomek A, Mikulik R, Magerova H, Horinek D and Hort J: Neurosonological examination: A non-invasive approach for the detection of cerebrovascular impairment in AD. Front Behav Neurosci 8: 4, 2014.

17. Tachibana H, Washida K, Kowa H, Kanda F and Toda T: Vascular function in Alzheimer's disease and vascular dementia. Am J Alzheimers Dis Other Demen 31: 437-442, 2016.

18. Heinzel S, Liepelt-Scarfone I, Roeben B, Nasi-Kordhishti I, Suenkel U, Wurster I, Brockmann K, Fritsche A, Niebler R, Metzger FG, et al: A neurodegenerative vascular burden index and the impact on cognition. Front Aging Neurosci 9: 161, 2014.

19. McKhann G, Drachman D, Folstein M, Katzman R, Price D and Stadlan EM: Clinical diagnosis of Alzheimer's disease: Report of the NINCDS-ADRDA work group under the auspices of department of health and human services task force on Alzheimer's disease. Neurology 34: 939-944, 1984.

20. Hughes CP, Berg L, Danziger WL, Coben LA and Martin RL: A new clinical scale for staging of dementia. Br J Psychiatry 140 566-572, 1982

21. Román GC, Tatemichi TK, Erkinjuntti T, Cummings JL, Masdeu JC, Garcia JH, Amaducci L, Orgogozo JM, Brun A, Hofman A, et al: Vascular dementia: Diagnostic criteria for research studies. Report of the NINDS-AIREN International Workshop. Neurology 43: 250-260, 1993.

22. Hachinski VC, Iliff LD, Zilhka E, Du Boulay GH, McAllister VL, Marshall J, Russell RW and Symon L: Cerebral blood flow in dementia. Arch Neurol 32: 632-637, 1975.

23. Wahlund LO, Barkhof F, Fazekas F, Bronge L, Augustin M, Sjögren M, Wallin A, Ader H, Leys D, Pantoni L, et al: A new rating scale for age-related white matter changes applicable to MRI and CT. Stroke 32: 1318-1322, 2001.

24. Zhou HD, Wang JY, Li J, Deng J, Gao C and Chen M: Study on frequency and predictors of dementia after ischemic stroke: The Chongqing stroke study. J Neurol 251: 421-427, 2004.

25. Rosen WG, Mohs RC and Davis KL: A new rating scale for Alzheimer's disease. Am J Psychiatry 141: 1356-1364, 1984.

26. Fuld PA, Masur DM, Blau AD, Crystal H and Aronson MK: Object-memory evaluation for prospective detection of dementia in normal functioning elderly: Predictive and normative data J Clin Exp Neuropsychol 12: 520-528, 1990.

27. Zhang M: Prevalence study on dementia and Alzheimer disease. Zhonghua Yi Xue Za Zhi 70: 424-428, 430, 1990 (In Chinese).

28. Welsh KA, Butters N, Hughes JP, Mohs RC and Heyman A: Detection and staging of dementia in Alzheimer's disease. Use of the neuropsychological measures developed for the consortium to establish a registry for Alzheimer's disease. Arch Neurol 49: 448-452, 1992

29. Katz S, Downs TD, Cash HR and Grotz RC: Progress in development of the index of ADL. Gerontologist 10: 20-30, 1970.

30. Touboul PJ, Prati P, Scarabin PY, Adrai V, Thibout E and Ducimetière P: Use of monitoring software to improve the measurement of carotid wall thickness by B-mode imaging. J Hypertens 10 (Suppl): S37-S41, 1992.

31. Dubey AK, Varma Y, Dubey S, Singh G, Dubey AK, Sharma V and Grade S: PW195 lipid profile and carotid intimal medial thickness in different stages of chronic kidney disease. Global Heart 9: e297, 2014.
32. Xiang J, Zhang T, Yang QW, Liu J, Chen Y, Cui M, Yin ZG, Li L Wang YJ, Li J and Zhou HD: Carotid artery atherosclerosis is correlated with cognitive impairment in an elderly urban Chinese non-stroke population. J Clin Neurosci 20: 1571-1575, 2013.

33. Firebaugh G, Warner $C$ and Massoglia M: Fixed effects, random effects and hybrid models for causal analysis. In Morgan SL (ed). Handbook of Causal Analysis for Social Research. Springer Science+Business Media Dordrecht, pp113-132, 2013.

34. Schneider JA, Wilson RS, Bienias JL, Evans DA and Bennett DA: Cerebral infarctions and the likelihood of dementia from Alzheimer disease pathology. Neurology 62: 1148-1155, 2004.

35. Snowdon DA, Greiner LH, Mortimer JA, Riley KP, Greiner PA and Markesbery WR: Brain infarction and the clinical expression of Alzheimer disease: The Nun study. JAMA 227: 813-817, 1997.

36. Ruitenberg A, den Heijer T, Bakker SL, van Swieten JC, Koudstaal PJ, Hofman A and Breteler MM: Cerebral hypoperfusion and clinical onset of dementia: The Rotterdam Study. Ann Neurol 57: 789-794, 2005.

37. Toledo JB, Arnold SE, Raible K, Brettschneider J, Xie SX, Grossman M, Monsell SE, Kukull WA and Trojanowski JQ: Contribution of cerebrovascular disease in autopsy confirmed neurodegenerative disease cases in the National Alzheimer's Coordinating Centre. Brain 136: 2697-2706, 2013.

38. Stampfer MJ: Cardiovascular disease and Alzheimer's disease: Common links. J Intern Med 260: 211-223, 2006.

39. de la Torre JC: Is Alzheimer's disease a neurodegenerative or a vascular disorder? Data, dogma, and dialectics. Lancet Neurol 3: 184-190, 2004

40. Lathe R, Sapronova A and Kotelevtsev Y: Atherosclerosis and Alzheimer-diseases with a common cause? Inflammation, oxysterols, vasculature. BMC Geriatr 21: 14-36, 2014.

41. Devereux RB and Alderman MH: Role of preclinical cardiovascular disease in the evolution from risk factor exposure to development of morbid events. Circulation 88: 1444-1455, 1993.

42. Bots ML, van Swieten JC, Breteler MM, de Jong PT, van Gijn J, Hofman A and Grobbee DE: Cerebral white matter lesions and atherosclerosis in the Rotterdam study. Lancet 341: 1232-1237, 1993.

43. Viswanathan A, Rocca WA and Tzourio C: Vascular risk factors and dementia. How to move forward? Neurology $72: 368-374$, 2009.

44. Deschaintre Y, Richard F, Leys D and Pasquier F: Treatment of vascular risk factors is associated with slower decline in Alzheimer disease. Neurology 73: 674-680, 2009.

45. Morris JC, Storandt M, Miller JP, McKeel DW, Price JL, Rubin EH and Berg L: Mild cognitive impairment represents early-stage Alzheimer disease. Arch Neurol 58: 397-405, 2001.

46. Silvestrini M, Vernieri F, Pasqualetti P, Matteis M, Passarelli F, Troisi E and Caltaqirone C: Impaired cerebral vasoreactivity and risk of stroke in patients with asymptomatic carotid artery stenosis. JAMA 283: 2122-2127, 2000. 